\title{
Du parallèle dans la déclamation
}

\section{Danielle van Mal-Maeder}

\section{Q OpenEdition}

Journals

Édition électronique

URL : https://journals.openedition.org/rhetorique/1140

DOI : 10.4000/rhetorique. 1140

ISSN : 2270-6909

\section{Éditeur}

UGA Éditions/Université Grenoble Alpes

\section{Édition imprimée}

ISBN : 978-2-37747-296-3

\section{Référence électronique}

Danielle van Mal-Maeder, « Du parallèle dans la déclamation », Exercices de rhétorique [En ligne], 16। 2021, mis en ligne le 17 mai 2021, consulté le 19 mai 2021. URL : http://journals.openedition.org/ rhetorique/1140 ; DOI : https://doi.org/10.4000/rhetorique.1140

Ce document a été généré automatiquement le 19 mai 2021.

\section{(c) (i) (2)}

Les contenus de la revue Exercices de rhétorique sont mis à disposition selon les termes de la Licence Creative Commons Attribution - Pas d'Utilisation Commerciale - Partage dans les Mêmes Conditions 4.0 International. 


\title{
Du parallèle dans la déclamation
}

\author{
Danielle van Mal-Maeder
}

\section{Le parallèle comme exercice de rhétorique}

1 Dans l'Antiquité, l'exercice de la déclamation, suasoire et controverse, représentait le couronnement de l'apprentissage rhétorique. Avant de l'aborder, les élèves s'entraînaient avec une série d'exercices préparatoires, les progymnasmata ${ }^{1}$. Comme j'ai tenté de le montrer ailleurs, ces derniers entraient dans la composition d'une déclamation, que ce soit en se fondant dans sa structure (c'est le cas du récit dans la narratio, de la contestation et de la confirmation dans l'argumentatio) ou en donnant lieu à des amplifications (par exemple le lieu commun ou la description ${ }^{2}$ ). Parmi ces exercices, on trouve le parallèle (synkrisis en grec, comparatio en latin) que Théon définit sobrement comme « un discours qui compare le meilleur ou le pire» (Progymnasmata, $10,112)$, tandis qu'Aphthonios parle d'« un discours qui compare au moyen d'une opposition et dont la conclusion met le plus du côté du comparé » (Progymnasmata, $10,1)$. Suivent une énumération des sujets possibles, des exemples et la méthode à appliquer. Selon Théon, il existe deux façons de procéder : « ou bien nous proposons un développement particulier pour chacun des éléments mis en parallèle, ou bien nous n'en proposons qu'un seul pour les deux en donnant l'avantage à l'un des deux " (Progymnasmata, 10,115). Aphthonios recommande de ne pas faire un parallèle « ensemble contre ensemble, car cela est plat et non agonistique, mais point contre point, car c'est cela qui est agonistique » (Progymnasmata, 10, 3).

2 Le parallèle n'a pas toujours fait partie du programme des progymnasmata. PseudoHermogène nous informe que certains théoriciens le rattachaient au lieu commun ou à l'éloge pour amplifier les fautes et les qualités (Progymnasmata 8,1) 3 $^{3}$ Selon Michel Patillon, c'est «l'importance prise par ce type de discours dans les trois genres oratoires et dans la littérature en général » qui amena les rhéteurs à l'inclure dans la liste comme exercice à part entière ${ }^{4}$. De fait, Friedrich Focke a mis en évidence l'omniprésence de la synkrisis dans la littérature antique et ses liens avec les textes agonistiques, citant comme exemples, entre autres, le débat entre Eschyle et Euripide 
dans les Grenouilles d'Aristophane et le certamen Homeri et Hesiodi ${ }^{5}$. La critique littéraire antique est friande de ce genre de confrontations : que l'on songe à Quintilien et son catalogue d'auteurs grecs et latins dont il évalue les mérites, à Aper et Maternus se faisant l'avocat l'un de l'art oratoire, l'autre de la poésie chez Tacite, ou encore à AuluGelle comparant les vers d'Homère et leur traduction par Virgile ${ }^{6}$. Dans le domaine de la déclamation, on citera Sénèque le Père, qui répertorie les meilleures (et les pires) trouvailles des déclamateurs de son temps en les juxtaposant de manière à ce que ses fils puissent se former leur propre jugement ${ }^{7}$. Dans la préface du livre 4 , il développe plus longuement un parallèle entre deux déclamateurs, Asinius Pollion et Haterius, pour opposer leur attitude respective face à la mort de leur enfant. Le premier recommença à déclamer trois jours après. Le second ne se remit jamais de cette perte : des années après, quand il endossait le rôle d'un père endeuillé, il éclatait encore en sanglots, incapable de séparer la fiction des déclamations de la réalité 8

Le lien avec l'éloge et le blâme apparaît clairement dans ce processus comparatif, et cela explique peut-être pourquoi l'exercice du parallèle leur succède dans la liste des progymnasmata. De son côté, après avoir traité de l'éloge, Quintilien déclare :

Hinc illa quoque exercitatio subit comparationis, uter melior uterue deterior. Quae quamquam uersatur in ratione simili, tamen et duplicat materiam et uirtutum uitiorumque non tantum naturam, sed etiam modum tractat.

Vient ensuite un autre exercice : la comparaison; de deux personnes, quelle est la meilleure, quelle est la pire ? La méthode est analogue dans les deux cas, mais, ici, la matière est double, et, pour les vices et les vertus, on examine non seulement leur nature, mais leur degré 9 .

\section{Genus comparatiuum}

4 Le parallèle n'est pas pour autant réservé à la rhétorique épidictique. On pourrait d'ailleurs affirmer que tout discours impliquant une controverse ou cherchant à imposer un point de vue engage nécessairement une confrontation d'arguments (même de façon implicite) et donc une forme de comparaison. Dans le passage où il traite des premiers exercices chez le rhéteur, Quintilien relève à propos de la thèse qu'elle peut comporter un parallèle, comme dans l'exemple « si la vie de la campagne est préférable à celle des villes" ou "si le renom d'un jurisconsulte l'emporte sur celui d'un militaire » (Institution oratoire, II, 4, 24). Pour illustrer ce dernier sujet, il cite le discours Pour Muréna, où Cicéron développe une telle comparaison (contentio : §19) à l'avantage de son client. On observe là une conjonction des trois genres : insérée dans un discours judiciaire, une comparaison devenue sujet de délibération donne lieu à un développement de nature épidictique.

Lorsqu'il traite de l'état de la cause dans son ouvrage De l'invention, Cicéron distingue les causes simples des causes complexes, ces dernières impliquant parfois une comparaison. Les exemples qu'il cite appartiennent au genre délibératif :

Constitutione causae reperta statim placet considerare utrum causa sit simplex an iuncta; et, si iuncta erit, utrum sit ex pluribus quaestionibus iuncta an ex aliqua conparatione. Simplex est quae absolutam in se continet unam quaestionem, hoc modo: "Corinthiis bellum indicamus an non?" Coniuncta ex pluribus quaestionibus in qua plura quaeruntur, hoc pacto: "Vtrum Cartago diruatur an Cartaginiensibus reddatur an eo colonia deducatu ». Ex conparatione in qua per contentionem utrum potius aut quid potissimum quaeritur, ad hunc modum : «Vtrum exercitus in Macedoniam contra Philippum mittatur, 
qui sociis sit auxilio, an teneatur in Italia, ut quam maximae contra Hannibalem copiae sint ».

Dès que l'état de la cause a été trouvé, il convient de considérer si celle-ci est simple ou complexe; et si elle est complexe, de voir si elle est constituée de plusieurs questions ou d'une alternative. Est simple la cause qui comporte une seule question, faisant un tout. Exemple: «Déclarons-nous, oui ou non, la guerre aux Corinthiens?» Elle est complexe, avec plusieurs questions, quand on examine plusieurs points. Par exemple: "Doit-on détruire Carthage, la rendre aux Carthaginois, ou y conduire une colonie ?» Elle comporte une alternative quand on cherche, en comparant, si une chose est préférable à une autre ou à plusieurs autres. De cette manière: «L'armée doit-elle être envoyée en Macédoine contre Philippe pour secourir nos alliés ou doit-elle être gardée en Italie pour opposer les troupes les plus nombreuses possible à Hannibal ${ }^{10}$ ? »

On relèvera dans ce texte, outre les termes conparatio et contentio, le comparatif potius et le superlatif potissimum. Ces degrés de comparaison impliquent des critères permettant de guider la prise de décision. Ces critères, nous le verrons, font appel à l'utilitas (l'utilité), la necessitas (la nécessité), l'honestas (l'honnêteté), la dignitas (le mérite) et la iustitia (la justice). Quintilien distingue également, mais à propos du genre judiciaire, les causes simples des causes complexes, qui portent respectivement sur un ou plusieurs points de litige. Il évoque encore un autre type :

Diuersum his tertium genus, quod dicitur comparatiuum. Cuius rei tractatus in parte causae frequens est, ut cum apud centumuiros post alia quaeritur et hoc, uter dignior hereditate sit. Il existe un troisième genre de causes, différent des précédents, que l'on appelle comparatif. Il apparaît souvent dans une partie de cause, comme, par exemple, devant les centumvirs, lorsque, après d'autres questions, est soulevée celle de savoir quel est le plus qualifié de deux prétendants à un héritage ${ }^{11}$.

On trouve dans les Petites déclamations (ou Déclamations mineures) du Pseudo-Quintilien un cas correspondant à cet exemple. Ce recueil à usage scolaire, qui date probablement $\mathrm{du} \mathrm{I}^{\mathrm{er}}$ siècle de notre ère, contient des déclamations-modèles « en miniature " (ce ne sont parfois que des ébauches ou des parties de discours), exposant les différents points qu'il s'agit de développer. Certaines sont accompagnées d'un exposé théorique plus ou moins élaboré (le sermo du «Maître» du recueil) énonçant toutes sortes de recommandations ${ }^{12}$. L'une des déclamations met en scène trois frères, un orateur, un médecin et un philosophe se disputant l'héritage de leur père. Ce dernier avait en effet décidé de léguer ses biens à celui des trois qui se montrerait le plus utile à la collectivité :

Orator medicus philosophus. Contendunt orator, medicus, philosophus de bonis patris, qui testamento eum heredem reliquerat qui se probasset amplius prodesse ciuibus.

L'orateur, le médecin et le philosophe. Un orateur, un médecin et un philosophe se disputent les biens de leur père, qui, dans son testament, avait désigné comme héritier celui qui se serait montré le plus utile pour ses concitoyens ${ }^{13}$.

Cette controverse s'inscrit dans une tradition de synkrisis bien spécifique, la dispute entre artes : outre les exemples mentionnés plus haut (jurisprudence versus art militaire chez Cicéron ; art oratoire versus poésie chez Tacite), on citera celle qui oppose l'art du cuisinier et celui du boulanger chez Vespa, dont Emanuele Berti a montré qu'elle est une controverse en vers ${ }^{14}$. Chez le Pseudo-Quintilien, c'est le médecin qui prononce le discours. Il démontre d'abord l'inutilité de la philosophie, puis celle de l'éloquence (selon lui, elle ne vise pas le juste et peut même se révéler nuisible ${ }^{15}$ ), après quoi il leur oppose les mérites de sa propre discipline. Ici, le parallèle se développe autour de la notion d'utilitas, ensemble contre ensemble, en recourant au blâme et à l'éloge pour faire triompher la cause de la médecine. 
7 Les connaissances d'un médecin en matière de potions peuvent en effet se révéler très utiles pour se débarrasser d'un tyran, comme le montre ce sujet de controverse cité par Quintilien, qui implique également une argumentation fondée sur la comparaison :

Est et alia duplex coniectura, huic anticategoriae diuersa, de praemiis, ut in illa controuersia : "Tyrannus suspicatus a medico suo datum sibi uenenum torsit eum, et cum is dedisse se pernegaret arcessit alterum medicum: ille datum ei uenenum dixit, sed se antidotum daturum, et dedit potionem ei, qua epota tyrannus decessit. De praemio duo medici contendunt». Nam ut illic factum in aduersarium transferentium, ita hic sibi uindicantium personae, causae, facultates, tempora, instrumenta, testimonia comparantur. Il y a aussi un autre type de causes conjecturales qui comportent deux questions, mais diffèrent de l'anticategoria ; c'est quand il s'agit de récompenses, comme dans la controverse suivante: «Un tyran, ayant soupçonné son médecin de lui avoir donné du poison, le mit à la torture, et, comme il persistait à nier, le tyran fit venir un autre médecin ; celui-ci assura qu'on lui avait administré du poison, mais qu'il lui donnerait un antidote; il donna une potion; le tyran la but et mourut. Il y a contestation entre les deux médecins à propos de la récompense ». Ici, comme dans les causes où il y a rejet de l'accusation sur l'adversaire, on compare, à propos de ceux qui revendiquent une récompense, les caractères, les motifs, les moyens, les circonstances, les instruments, les témoignages ${ }^{16}$.

Le tyrannicide est un personnage type de l'univers déclamatoire. Il appartient à la famille des figures (en principe) positives; le fait d'avoir tué un tyran lui donne droit à une récompense ${ }^{17}$. Ici, deux médecins se disputent cet honneur, chacun affirmant avoir empoisonné le tyran. Le motif de la récompense réclamée par deux personnes (ou plus) entre dans la catégorie du genus comparatiuum. Quintilien relève qu'un sujet de ce genre donne lieu à différentes comparaisons (personnes, motifs, moyens, temps, instruments, témoignages) sans en préciser les critères. Qu'est-ce donc qui permet de décider auquel des deux médecins attribuer le mérite d'avoir empoisonné le tyran? L'un des critères est la dignitas, comme on peut le déduire de cet autre passage, où Quintilien mentionne à nouveau l'exemple du tyrannicide :

De praemiis autem quaeruntur duo: an ullo sit dignus qui petit, an tanto; ex duobus, uter dignior, ex pluribus, quis dignissimus. Quorum tractatus ex ipso meritorum genere ducuntur. De praemiis autem quaeruntur duo: an ullo sit dignus qui petit, an tanto; ex duobus, uter dignior, ex pluribus, quis dignissimus. Quorum tractatus ex ipso meritorum genere ducuntur.

Quand il s'agit, d'autre part, de récompenses, il y a deux points à examiner: le candidat en mérite-t-il une? en mérite-t-il une aussi importante; s'il y a deux candidats, qui est plus méritant, s'ils sont plusieurs, quel est le plus méritant. Ces points se traitent en considérant la nature même des services ${ }^{18}$.

8 Cette question est examinée à propos du tyrannicide dans deux autres Petites déclamations pseudo-quintiliennes. Un riche et un pauvre (meilleurs ennemis du monde déclamatoire) s'y disputent la récompense, le premier ayant engagé le deuxième pour assassiner le tyran de la ville ${ }^{19}$. Dans la déclamation 345 , le pauvre compare l'action de son adversaire à la sienne :

Tu mihi dicis: "Pecuniam dedi et ex otiosa iacente patrimonii parte aliquid numeraui", cum ego dicam: "Arcem conscendi, satellites straui, nefarium corpus repetitis ictibus trucidaui $»$.

Toi, tu me dis : «J'ai donné de l'argent, j'ai pris quelque chose sur une partie de mes biens qui sommeillaient paisiblement», alors que moi je dirai : "J'ai escaladé la citadelle, j'ai terrassé les sentinelles, j'ai transpercé ce corps maudit de multiples $\operatorname{coups}^{20} »$.

Le parallèle se construit sur une opposition, qui vise à discréditer l'action du riche (son inaction, plutôt : cf. le redoublement "otiosa iacente... parte ») et à louer l'exploit du 
pauvre. Les prétentions des deux adversaires, citées au discours direct, sont introduites par le verbe dico (dicis/dicam) et le pronom personnel correspondant ( $t u / e g o)$. La comparaison est appuyée par une construction grammaticale en reprise avec crescendo et par les terminaisons des verbes (pecuniam dedi/arcem conscendi, stallites straui ; aliquid numeraui/nefarium corpus... trucidaui).

D'autres personnages types de l'univers déclamatoire se disputent une récompense : les héros de guerre, auxquels une loi accorde la possibilité d'émettre n'importe quel souhait en remerciement de leur engagement ${ }^{21}$. L'ajout d'une deuxième loi (ou d'un article de loi) dans le thème d'une controverse amène à développer un parallèle. C'est le cas dans la Petite déclamation 258, où un père et un fils s'affrontent en fonction de ce principe: "Si duo aut plures fortiter fecerint, de praemio armis contendant» ("Si deux hommes ou plus ont combattu héroïquement, ils se disputeront la récompense par les armes »). Le père demande à son fils de lui céder la place, sans doute pour éviter d'avoir à se battre avec lui. Devant son refus, c'est lui qui renonce à la récompense, après quoi il chasse son enfant. Cette controverse aborde une thématique qui obsède la déclamation antique, à savoir le conflit entre père et fils, lié à la question de la patria potestas, de l'autorité paternelle. Dans ce contexte, elle est envisagée de manière à établir une comparaison entre deux héros appartenant à des générations différentes, puisque l'un est un senex (un vieillard), l'autre un iuuenis (un jeune homme). Le déclamateur, qui endosse le rôle du père, estime ainsi qu'en raison de son âge, il a mieux mérité que son fils; sa valeur (uirtus) le rend plus digne de la récompense :

Age uero, non praeter hoc etiam dignior praemio fuit uirtus mea? Non est tam admirabile cum fortiter facit iuuenis. Hoc aetas postulat, hoc robur iuuentae poscit; paene turpe est non fecisse. Hoc est honore dignius, uicisse annos et ultra aetatem durantes agere uirtutes. Eo tempore fortiter feci quo cogi non possem ut militarem. Vtrum tandem uis magnum esse quod ego feci an minus esse quod tu fecisti?

Mais allons, en dehors de cela, est-ce que ma valeur ne m'a pas rendu plus digne d'une récompense ? Il n'y a rien de tellement surprenant à ce qu'un jeune combatte héroïquement. C'est ce qu'exige son âge, ce que la force de sa jeunesse réclame; il serait presque honteux de ne pas l'avoir fait. Ce qui est plus honorable, c'est d'avoir triomphé des années et d'agir en héros bien au-delà de la limite d'âge. Je me suis battu avec bravoure à un moment où on ne pouvait pas m'obliger à servir. Alors selon toi : ce que j'ai fait est grand ou ce que tu as fait est plus petit ${ }^{22}$ ?

La Petite déclamation 278 propose une variante pour honorer le père d'un héros de guerre : «Viri fortis pater decem milia accipiat » (« Le père d'un héros de guerre recevra dix mille sesterces »). Ici, le uir fortis, le héros de guerre, est un enfant exposé, dont le père biologique ressurgit pour réclamer les dix mille sesterces au père adoptif. $\mathrm{Ce}$ dernier refuse, en développant un parallèle sous l'angle de l'aequitas (équité). Lui aussi affirme être le plus digne (dignior) de recevoir la récompense :

Reliquum est ut intueamur uter nostrum hac pecunia dignior esse uideatur. Et ut breuiter dicam, tu exposuisti, ego sustuli. Scio te coepturum altius, ut dicas : "Genui ». Hoc est quare tibi debeamus irasci : quantum ad te pertinet, non habet respublica uirum fortem; aut illum ferae lacerauerunt aut aues diripuerunt aut aliquis (quod multo sit indignius) sustulit leno aut lanista. Ex meis ille beneficiis natus est.

Il reste à examiner lequel d'entre nous semble être le plus digne de cet argent. Et pour le dire en deux mots, toi tu l'as exposé, moi je l'ai recueilli. Je sais bien, tu vas remonter plus haut en disant : «Je l'ai mis au monde ». Voilà pourquoi nous devons être en colère contre toi : si cela n'avait tenu qu'à toi, l'État n'aurait pas eu de héros de guerre ; il aurait été mis en pièce par des bêtes sauvages ou alors déchiqueté par des rapaces ou encore - et cela est bien plus ignoble - il aurait été recueilli par un maquereau ou un entraîneur de gladiateurs ${ }^{23}$. 
Une brève sentence fondée sur un parallèle («tu exposuisti, ego sustuli », «toi tu l'as exposé, moi je l'ai recueilli ») oppose l'action du père biologique à celle du père adoptif : ce dernier a élevé l'enfant que l'autre avait abandonné ${ }^{24}$. Ce constat est ensuite développé en une amplification de tonalité pathétique, qui anticipe un argument de la partie adverse fondé sur la temporalité («j'étais son père avant toi »). Sans lui, affirme le père adoptif, l'enfant serait mort ou aurait connu un sort infâme; sans lui, l'État n'aurait pas bénéficié des services de celui qui allait devenir un uir fortis. Ici, comme dans les cas où deux héros de guerre ou deux tyrannicides réclament la récompense, la loi entre en conflit avec elle-même. Pour ce genre de situation, Quintilien recommande d'examiner quelle demande a la priorité du point de vue temporel (« utra prior sit? ») et laquelle est la plus juste (« utra iustior sit petitio?»), ce qui implique une comparaison ${ }^{25}$.

\section{Lois contradictoires}

11 Il arrive fréquemment que deux lois gouvernent un thème de controverse et qu'elles se contredisent. Une comparaison permet alors de déterminer laquelle l'emporte. Un passage de Cicéron nous éclaire sur ce point à propos d'une affaire historique à caractère romanesque :

Ex contrariis autem legibus controuersia nascitur, cum inter se duae uidentur leges aut plures discrepare, hoc modo : lex: "Qui tyrannum occiderit Olympionicarum praemia capito et quam uolet sibi rem a magistratu deposcito et magistratus ei concedito $\%$. Et altera lex: "Tyranno occiso, quinque eius proximos cognatione magistratus necato ». Alexandrum, qui apud Pheraeos in Thessalia tyrannidem occuparat, uxor sua, cui Thebe nomen fuit, noctu, cum simul cubaret, occidit. Haec filium suum, quem ex tyranno habebat, sibi in praemii loco deposcit. Sunt qui ex lege occidi puerum dicant oportere. Res in iudicio est. In hoc genere utramque in partem idem loci atque eadem praecepta conuenient, ideo quod uterque suam legem confirmare, contrariam infirmare debebit. Primum igitur leges oportet contendere considerando utra lex ad maiores, hoc est ad utiliores, ad honestiores ac magis necessarias res pertineat.

Le débat naît des lois contradictoires, quand deux lois - ou davantage - semblent en désaccord. Exemple: soit une loi : «Le meurtrier d'un tyran recevra les mêmes récompenses que les vainqueurs aux jeux olympiques; il demandera au magistrat ce qu'il voudra et le magistrat le lui donnera ». Et cette autre loi : "Après la mort d'un tyran, le magistrat fera tuer ses cinq plus proches parents par le sang». Alexandre avait pris le pouvoir à Phères en Thessalie : une nuit, sa femme, appelée Thébé, qui couchait à ses côtés, le tua. Elle réclame à titre de récompense le fils qu'elle avait eu du tyran. Mais certains disent que, selon la loi, il faut tuer l'enfant. L'affaire passe en jugement. Dans ce type de cause les mêmes lieux et les mêmes préceptes conviendront aux deux parties, parce que chacun devra défendre la loi qui lui est favorable et ruiner celle qui la contredit. Il faut donc d'abord comparer les deux lois en examinant quelle est celle qui concerne les choses les plus importantes, c'est-à-dire les plus utiles, les plus honorables et les plus nécessaires ${ }^{26}$.

D'après ce texte, les critères sur lesquels se fonde la comparaison des lois sont l'utilitas, l'honestas et la necessitas. Une liste similaire se retrouve dans la Petite déclamation 274, où une confrontation entre lois donne lieu à un véritable casse-tête. Le sujet est le suivant : un tyran est frappé par la foudre en plein forum. Que faut-il faire de son corps? La question se pose en raison de deux lois citées dans le thème : 1. "Quo quis loco fulmine ictus fuerit, eodem sepeliatur " ("Là où un homme aura été frappé par la foudre, à cet endroit même, il faudra l'ensevelir »); 2. "Tyranni corpus extra fines abiciatur» ("Le cadavre d'un tyran sera jeté hors des frontières»). Ici, le Maître du recueil livre une ébauche de plaidoyer pour les deux parties. Chacune est précédée d'un court exposé 
théorique (le sermo), centré sur la comparaison des lois. Comme le relève Giuseppe Dimatteo, le Maître y présente en parallèle les arguments de l'une et l'autre partie ${ }^{27}$. Dans le premier sermo, il déclare :

In legum comparatione multa quaeri possunt, sed uarie in quaque controuersia. Interim quaeri solet an priuata altera, altera publica sit, et utrum militaris altera, altera pertinens ad ciuilia officia. Hic omnia fere cetera paria sunt ; utra utilior et magis necessaria ciuitati sit quaerendum est.

Dans une comparaison de lois, on peut soulever beaucoup de questions, différentes d'une controverse à l'autre. Parfois, on se demande si l'une est privée, l'autre publique, si l'une est militaire, tandis que l'autre concerne les devoirs civiques. Dans le cas présent, tout le reste à peu près se vaut; il faut alors examiner laquelle des deux lois est la plus utile et la plus nécessaire à la communautée ${ }^{28}$.

Ces critères sont illustrés dans le plaidoyer recommandant l'interdiction d'ensevelir le tyran. Le déclamateur propose d'abroger la première loi, car, contrairement à la deuxième, elle n'est pas utile :

Fulmine icti ut eodem loco sepeliantur quo sunt percussi ad quam tandem ciuitatis pertinet utilitatem? At hercule ut insepultus abiciatur tyrannus ad uindictam, ad securitatem pertinet. Non satis putauerunt maiores eas poenas aduersus tyrannum constituere quas possit excipere in uita. Multos magis tangit sepultura; ad cogitationem post se futurorum plerique grauius mouentur. Vultis scire? Propter hoc litigatur. Est ergo utilior.

Ensevelir les personnes frappées par la foudre à l'endroit même où elles ont été touchées, quel est l'avantage pour la communauté, je vous le demande? Alors qu'un tyran abandonné sans sépulture, pardi! voilà qui répond à la vengeance, à la sécurité. Nos ancêtres ont estimé qu'il ne suffisait pas de prévoir contre un tyran des peines qu'il pourrait encourir de son vivant. Bien des gens se soucient davantage de leur sépulture, la plupart sont plus profondément touchés à la pensée de ce qui leur arrivera après leur mort. Vous voulez savoir ? C'est pour cette raison que nous avons ce débat. Par conséquent, cette mesure est plus utile ${ }^{29}$.

Dans la pars altera (la partie adverse), le Maître développe un autre critère de comparaison, fondé cette fois sur le religieux, en faveur de la première loi. Considérant la foudre comme un châtiment divin, il soutient que cette loi a été rédigée pour satisfaire non pas les hommes, mais les dieux :

Neque hoc propter personam neque in honorem alicuius scripsisse potest legum lator uideri, sed in reuerentiam deorum. Fas non est uisum mouere ictum illum: quomodo sederant flammae, ita concremandum corpus uniuersum putauerunt. Existimatis locum illum corpori datum ? Fulmini datus est.

On ne peut pas croire que le législateur a rédigé cette loi pour une personne ou en l'honneur de quelqu'un, non: c'est par respect envers les dieux. Il a semblé sacrilège de déplacer l'individu frappé par la foudre: ils ont estimé qu'il fallait entièrement brûler le corps là où les feux s'étaient abattus. Vous pensez que cet endroit a été assigné au corps ? Il a été assigné à la foudre ${ }^{30}$.

13 Le critère religieux est également présent dans la Petite déclamation 324, qui confronte deux autres lois : 1. «Qui reum caedis damnauerit, bona eius possideat» («Celui qui aura fait condamner un homme accusé de meurtre prendra possession de ses biens»); 2. «Sacrilegi bona templo consecrentur» («Les biens d'un sacrilège seront consacrés au temple qu'il a dévalisé »). Dans cette affaire, un homme accusé de meurtre avoue sous la torture avoir également commis un sacrilège. Son accusateur et le prêtre du temple profané se disputent ses biens en s'appuyant respectivement sur la première et la deuxième loi. Le discours qui suit l'énoncé du thème est celui du prêtre. Il commence par une comparaison de personnes, de manière à opposer la noblesse de ses motivations à celles de son adversaire, qu'il dépeint comme un délateur cupide : 
Antequam leges comparamus, intueri personas libet. Nos nullam habemus in litigando propriam cupiditatem : non adquirimus nobis, petimus bona templo, petimus honorem deo. Aduersarius sibi uindicat et in praemium operae suae deposcit; ostendit quare accusauerit. Avant de comparer les lois, j'aimerais examiner les personnes. Nous, dans ce litige, nous n'avons aucun intérêt personnel : nous ne cherchons pas à nous enrichir, nous réclamons ces biens pour le temple, nous réclamons que l'honneur du dieu soit rétabli. Quant à notre adversaire, il les revendique pour lui-même et il les exige comme récompense pour son action; il dévoile ainsi pourquoi il s'est porté accusateur $^{31}$.

Ici, le parallèle permet de blâmer l'adversaire. Il précède une comparaison des lois, le prêtre y affirmant la primauté de la deuxième, en raison de son caractère sacré, d'une part, mais aussi parce que le vol a, d'après lui, précédé le meurtre : ce critère temporel forme ensuite l'essentiel de son argumentation (§ 5-7).

\section{Parallèles en contexte}

Examinons maintenant de plus près le fonctionnement du parallèle à l'intérieur du discours, en prenant l'exemple de trois Grandes déclamations pseudo-quintiliennes. Ce recueil (il s'agit probablement d'une anthologie à usage scolaire) contient dix-neuf controverses complètes d'époques différentes, qui offrent prise à une analyse du discours d'ensemble. La première est intitulée «Le mur aux traces de main ensanglantée ». Elle oppose un jeune aveugle et sa belle-mère, s'accusant réciproquement d'avoir assassiné le père de famille. Ce dernier avait été retrouvé mort dans son lit, aux côtés de son épouse endormie, l'épée de son fils - et héritier - plantée dans sa poitrine. Sur le mur, des taches de sang conduisaient à la chambre du garçon. Le discours qui suit l'énoncé du thème est celui de l'avocat du fils ${ }^{32}$.

À propos de ce genre d'affaire, où une défense s'accompagne d'une contre-accusation (antikategoria, mutua ou concertatiua accusatio en latin), Quintilien remarque qu'elle nécessite une comparaison de personnes, de motifs et d'autres éléments ${ }^{33}$. Bien que l'avocat du fils aveugle prétende vouloir se passer d'une comparaison de personnes ${ }^{34}$, son plaidoyer est construit sur un parallèle, ensemble contre ensemble, pour examiner deux questions : 1. Comment le crime a-t-il été commis? 2. À qui profite-t-il? Dans un premier temps, il défend son client en s'appuyant sur son handicap : il est impossible et tout à fait invraisemblable, affirme-t-il, que l'aveugle ait commis ce crime - qui plus est avec sa propre arme - et qu'il l'ait signé en laissant des empreintes ensanglantées sur son passage ; de plus, il n'avait aucun motif de tuer son père. Dans un deuxième temps, l'avocat reconstitue la scène de crime : c'est la marâtre qui aurait dérobé l'épée de son beau-fils, tué son mari alors qu'il était endormi à côté d'elle et disposé de fausses preuves pour faire inculper l'héritier (susceptible d'être condamné à mort pour parricide). Les mêmes éléments sont donc examinés - essentiellement selon le critère de la vraisemblance - dans la défense et dans l'accusation, qui s'étendent entre la narratio (le récit des faits) et la confirmatio (la confirmation) ${ }^{35}$. Dans la peroratio, partie du discours faisant une large place au pathos, l'avocat développe encore un parallèle, qui n'est autre qu'une comparaison de personnes :

Reliquum est ut intueamur, ille qui periit, ab utro magis uestrum desideretur. Te, opinor, hic grauius afficit dolor, inpatientius hic luctus exanimat, te, quae absoluta protinus nubes, et tempori accommodata lugubria flammeo reuertente mutabis. At hic uero iuuenis, qui, si fortunae suae mala cum praeteritis comparet, caecus coepit esse nunc primum, quid non miser in hoc sene perdidit! Viuebat illi magna pietas, aderant, quodcumque iusserat, de 
facie patris oculi : non inludere infelicibus tenebris contumaces seruuli poterant, nec, quod extremum contumeliarum genus est, ut dominum ageret rogabant.

Il nous reste à examiner lequel d'entre vous deux regrette le plus l'homme qui est mort. C'est toi, je suppose, que cette douleur tourmente plus durement, que ce deuil épuise plus cruellement, toi qui, une fois acquittée, te remarieras sans attendre et échangeras ces vêtements de deuil que tu as endossés exprès pour la circonstance contre un nouveau voile nuptial. Ce garçon, en revanche, s'il compare les maux de son sort actuel aux précédents, c'est maintenant qu'il commence à être aveugle. Le pauvre, que n'a-t-il pas perdu avec le vieil homme! Quand son père était en vie, il avait droit à beaucoup d'affection; quel que soit l'ordre qu'il avait donné, des yeux étaient là pour lui, ceux de son père: les esclaves effrontés ne pouvaient pas rire aux dépens de sa triste cécité, et ils ne lui demandaient pas - ce qui est la pire des insultes - de jouer au maître de maison ${ }^{36}$.

Ce parallèle, qui prétend comparer les sentiments de la marâtre et ceux de son beaufils, n'a pas pour fonction de soutenir l'argumentation, mais de susciter l'indignation des juges envers la première et leur pitié à l'égard du deuxième. L'avocat interpelle d'abord la belle-mère en prédisant son remariage avec une insistance remplie d'ironie ("Te... hic grauius afficit dolor, inpatientius hic luctus exanimat, te... lugubria... mutabis» : on notera le passage au temps du futur). À l'avenir de cette veuve joyeuse, il oppose la situation du jeune aveugle ("At hic uero... » : on notera le passage à troisième personne). Le parallèle se fait alors narratif, c'est-à-dire qu'il se déploie en une évocation de la vie passée du jeune homme, lorsqu'il pouvait encore compter sur le soutien de son père. Outre la recherche du pathétique, il répond à la question À qui profite le crime?, en démontrant qu'il n'était pas utile (critère de l'utilitas) à l'aveugle de se débarrasser de son protecteur.

Les déclamations où il est question d'un choix ou d'un dilemme et qui, par conséquent, contiennent un élément délibératif, mobilisent volontiers le parallèle. Ainsi dans la Grande déclamation V, intitulée "le malade racheté ", dont le sujet est le suivant : un père avait deux fils, l'un vertueux (frugi), l'autre débauché (luxuriosus). Capturés par des pirates, les jeunes gens écrivirent à leur père pour qu'il les rachète. Ce dernier vendit tous ses biens et se rendit chez les pirates. Jugeant insuffisante la rançon qu'il avait apportée, les bandits le contraignirent à choisir un de ses enfants. Le père trancha en faveur de son fils débauché, tombé malade durant sa captivité ; celui-ci ne tarda pas à mourir. Le fils vertueux réussit à s'enfuir. De retour à la maison, il refusa d'entretenir son père. Le discours qui nous est parvenu est celui du père plaidant sa cause ${ }^{37}$. L'argument principal qu'il avance pour justifier son choix est évidemment la maladie : il se devait de sauver le plus faible de ses enfants, sachant que l'autre avait de bonnes chances de s'échapper grâce à sa force physique et mentale. Cet argument se déploie dans deux parallèles. Le premier se situe dans la narratio. Sur un ton posé, le père y compare le caractère de ses fils (comparatio personarum), pour instruire les juges des éléments de l'affaire et renforcer la plausibilité des faits ${ }^{38}$ :

Hic namque robustus ac patiens, non molliri prosperis facilis, non accidentibus frangi, et quem de uoluptatium gaudiorumque contemptu scires parem quandoque fortuitis; traxerat ex firmitate mentis magnam protinus et in membra constantiam. Ille uero pariter in laetitiam metusque resolutus, alienus a curis, sollicitudinibus impar, delicatus, impatiens, et iam similis aegro.

Car celui qui se tient devant vous était fort et endurant, il ne se laissait pas facilement amollir par le succès, ni briser par le malheur ; son mépris des plaisirs et des jouissances attestait de sa constance face aux hasards que la vie nous réserve parfois. De sa fermeté d'esprit, il avait en même temps gagné une grande vitalité physique. L'autre, en revanche, s'abandonnait autant à l'exultation qu'aux peurs, 
indifférent aux soucis, et, face aux angoisses, instable, fragile, faible, semblable,

déjà, à un homme malade ${ }^{39}$.

Dans ce parallèle antithétique que l'on qualifiera de descriptif, la première partie («Hic namque... ») concerne le fils vertueux. Elle s'apparente à un éloge. La deuxième phrase ( Ille uero... ») se rapporte au fils débauché. Le père doit éviter de trop le blâmer, sinon il pourrait sembler incompréhensible qu'il l'ait préféré à son frère. Par conséquent, ses défauts sont dépeints avec une certaine indulgence et présentés comme la cause de sa faiblesse physique, l'origine de sa maladie ${ }^{40}$. On relèvera le paradoxe : la supériorité du fils vertueux justifie qu'il n'ait pas été choisi. Un autre parallèle intervient en faveur du malade dans l'argumentatio (l'argumentation). Il a cette fois pour but d'émouvoir. Il fait suite à une longue description de la caverne insalubre des brigands ${ }^{41}$ :

Compara, si uidetur, huic aegro captiuitatem tantum tuam. Tu quereris quod cibos pirata non praestet, ille remittit oblatos. Te nuda humus, nudum cubile frangit, ille ad singulos ardentis corporis motus in sua [supra] uincla uersatur et, quocumque membra lassata dolore transtulerit, in supplicium redit renouata patientia.

Compare, si tu veux bien, ta simple captivité à celle de cet homme malade. Toi, tu te plains de ce que le pirate ne te donne pas à manger; lui, il repousse ce qu'on lui apporte. Toi, le sol nu, une couche nue t'éreintent ; lui, à chaque mouvement de son corps brûlant, il roule sur ses entraves, et à peine a-t-il ravivé sa résistance en déplaçant ses membres épuisés par la douleur d'un côté ou de l'autre que son calvaire recommence ${ }^{42}$.

L'effet pathétique de cet autre parallèle antithétique provient de ce que le déclamateur s'adresse directement à son adversaire, en alternant chaque membre de la comparaison (Tu..., ille...; Te..., ille...) et en employant le présent historique, renforçant ainsi le caractère visuel de la scène ${ }^{43}$. Ce parallèle permet de justifier le choix du père : il ne pouvait faire autrement que de racheter son enfant mourant. Le critère sous-jacent est celui de la necessitas, que le déclamateur invoque tout au long de son discours ${ }^{44}$. Au moment de ses délibérations, le père avait également considéré le juste et l'utile : il était juste de racheter son fils mourant, inutile de racheter celui qui pouvait se sauver par lui-même - ce que la suite des événements avait confirmé ${ }^{45}$.

Autres pirates, autre dilemme, autre conflit familial : dans la Grande Déclamation VI, intitulée «Le cadavre rejeté sur la plage ", un mari et sa femme se disputent à propos de leur fils décédé. Capturé par des pirates, le père avait écrit chez lui pour demander qu'on le rachète. Le fils partit s'offrir en échange de son père, en dépit de l'interdiction de sa mère, devenue aveugle à force de pleurer. Il mourut sous les chaînes. Son corps, jeté dans la mer, échoua sur le rivage de sa patrie. Alors que son père veut lui rendre les honneurs funèbres, la mère s'y oppose en s'appuyant sur la loi suivante: "Qui in calamitate parentes deseruerit, insepultus abiciatur» («Celui qui aura abandonné ses parents dans le malheur, sera abandonné sans sépulture »). Dans son plaidoyer, le père explique que son fils a désobéi à sa mère pour pouvoir lui porter secours. Comme l'a relevé Thomas Zinsmaier, son argumentation est largement fondée sur la comparaison $^{46}$. Prenant appui sur le critère de la necessitas, il commence par rappeler la supériorité de la patria potestas (l'autorité paternelle) sur toute loi. Son fils n'avait pas le choix, il devait lui obéir :

Poteram quidem fortiter dicere: «Pater iussi. Hoc nomen omni lege maius est ; [...] Si non fecerit quod iubeo, non deferam illum ad sepulturam ». Necesse habuit parere : non deseruit, sed abductus est.

J'aurais pu recourir à un argument de force: «Moi, son père, je lui ai donné un ordre. Ce titre vaut plus que n'importe quelle loi; [...] S'il ne fait pas ce que 
j'ordonne, je ne permettrai pas qu'il soit enseveli. » Il lui fallait m'obéir : il ne t'a pas abandonnée, non, il a été emmené de force ${ }^{47}$.

Feignant de renoncer à la ligne argumentative qui affirme la supériorité des pères sur les mères ${ }^{48}$, il poursuit en invoquant le critère temporel. Puisqu'il avait été fait prisonnier avant que sa femme ne devienne aveugle, il avait la priorité :

Tempore certe uinco : ante miser esse coepi. Tu adhuc integra es, ego iam alligatus sum; tu sana in domo, ego iam paene defunctus in carcere; tibi adhuc non opus est filio, ego iam rogo. Nempe calamitas tua nata est post epistolam meam. Nisi te flentem consolari filius uoluisset, ante caecitatem tuam exisset. Noli mirari, si te gratia uinco : ante exoraui filium, quam tu rogares.

Du point de vue chronologique, il n'y a pas de doute, je l'emporte: mon malheur précède le tien. Quand pour toi, tout va bien encore, moi, je suis déjà couvert de chaînes; toi, tu es saine et sauve à la maison, moi je suis presque mort en prison ; toi, tu n'as pas encore besoin de ton fils, moi je fais déjà appel à lui. Car oui, ton malheur est survenu après que tu as reçu ma lettre. Si notre fils n'avait pas voulu te consoler quand tu te lamentais, il serait parti avant que tu ne deviennes aveugle. Ne t'étonne pas si je l'emporte en sollicitant ses faveurs : j'ai supplié notre fils avant que tu ne lui demandes de rester ${ }^{49}$.

Le parallèle est à nouveau stylistiquement très marqué, avec de courtes périodes antithétiques (on relèvera ici encore l'emploi du présent historique), l'anaphore des pronoms personnels (Tu..., ego; tu..., ego; tibi..., ego) et des répétitions martelant l'avantage du père (uinco... uinco ; ante... ante...). Il précède une longue comparaison des malheurs du mari et de sa femme, ensemble contre ensemble, dont la conclusion est la suivante: perdre la vue n'est rien en regard d'un enlèvement par des pirates. La première partie de cette comparaison est consacrée aux épreuves subies par la mère. Elle inclut un autre parallèle (parallèle enchâssé), qui compare plus généralement la condition des hommes et des femmes. Selon le déclamateur, devenir aveugle est moins grave pour une femme que pour un homme :

Quamquam cuiuslibet et quacumque causa hunc incursum passae leuius est tamen malum

feminae: non enim nauigatis, non legationem obitis, non frequenti peregrinatione uariatis aspectus, non militaris uos, non forensis ratio deducit. Alioquin semper estis intra domum, uno plurimum loco leuibus officiis adfixae.

Quelle que soit la personne et quelle qu'en soit la cause, ce malheur n'est pourtant jamais aussi léger que quand il touche une femme. Car vous ne naviguez pas, vous n'assumez pas de mission d'ambassade, vous ne changez pas d'horizon dans des voyages incessants, aucune affaire militaire, aucune affaire politique ne vous éloigne de chez vous. Du reste, vous demeurez toujours à l'intérieur de la maison, attachées à des tâches légères et le plus souvent à un seul endroit ${ }^{50}$.

La situation privilégiée des femmes (leur oisiveté, plutôt, soulignée par l'anaphore de la négation non) est dépeinte à travers une série de courtes périodes se référant par contraste aux multiples obligations des hommes. Ce parallèle est suivi de l'énumération des malheurs du mari, qui se déploie en une ample description détaillant l'attaque des pirates, les mauvais traitements subis, la sinistre caverne dans l'obscurité de laquelle il s'était retrouvé aussi aveugle que sa femme - mais obligé, lui, de supporter les cris des autres prisonniers ${ }^{51}$. Cette compétition de malheurs (contentio calamitatum) se termine par une synthèse, qui reprend tous les points mentionnés sous la forme d'oppositions binaires :

Compara terram mari, domum naui, lectulum carceri, libertatem seruituti, desiderium oculorum totius corporis damno. Quo nos longius ducit calamitatium nostrarum misera contentio ? Caeci etiam litigare possunt, captiui nec uiuere.

Compare la terre à la mer, une maison à un navire, un bon lit à une caverne, la liberté à la servitude, la perte des yeux à la déchéance d'un corps entier. Jusqu'où 
cette déplorable compétition de malheurs nous conduira-t-elle? Les aveugles peuvent même engager des procès, les prisonniers ne peuvent même pas survivre ${ }^{52}$.

Délaissant un instant les accents pathétiques, le déclamateur développe ensuite l'argument de l'utilitas : il était plus utile, déclare-t-il à son épouse, que leur fils vienne à son secours. Cette affirmation est réitérée dans une sentence construite sur un strict parallèle antithétique avec épiphore : « Ego redimi poteram, tu sanari non poteras » (« Moi, je pouvais être racheté, toi tu ne pouvais pas être guérie $\left.{ }^{53} »\right)$. Enfin, le plaidoyer inclut une autre forme de parallèle, érudite et emphatique, qui consiste à évoquer un personnage mythologique ou historique pour accentuer la gravité de son propre cas. Il s'agit (paradoxalement) d'un locus a minore ad maius (lieu du plus petit au plus grand) qu'on rencontre ailleurs dans les Grandes déclamations ${ }^{54}$. Au seuil de la peroratio, le déclamateur conclut ainsi l'éloge funéraire de son fils, mort à la place de son père, en comparant son acte héroïque à celui de deux figures légendaires, modèles de piété filiale :

Conparate nunc, si quis patrem per hostes solus tulit, qui, cum uenientia in patrem tela excepisset, simplici tamen morte defunctus est. Vnum legimus, qui uicarias pro patre non quidem piratis aut mari manus obtulerit, sed ubi redimi posset. Me miserum! Dicendum est quod hoc ipso admoneor exemplo: in honorem pietatis etiam damnati sepeliuntur.

Faites maintenant la comparaison avec ce héros qui, à lui seul, a porté son père au milieu des ennemis : s'il a recueilli les traits qui pleuvaient sur son père, il a malgré tout connu une mort sans complication. Dans les livres que nous lisons, un seul homme a tendu ses mains en échange contre son père - pas à des pirates ou sur la mer, non: dans un endroit où on pouvait le racheter. Pauvre de moi! Me voilà contraint de dire ce que cet exemple me rappelle : par respect pour leur père, même les condamnés sont ensevelis ${ }^{55}$.

Le premier membre de cette comparaison (doctement anonymisée) fait référence à Antiloque, fils de Nestor, qui sacrifia sa vie pour sauver son père pendant la guerre de Troie. Le deuxième concerne Cimon, dont le père Miltiade avait été condamné à une lourde amende en 489 avant notre ère et était mort peu après. Selon certaines sources, Cimon aurait pris sa place en prison, fait ensevelir son père, et aurait ensuite été racheté par sa sœur Elpinicé ${ }^{56}$. En hissant son fils au-dessus de ces exemples d'héroïsme et de piété, le père veut convaincre sa femme et les juges de l'ensevelir comme il se doit.

En résumé, le parallèle apparaît comme une forme discursive très flexible, adaptée aux trois genres de la rhétorique. Dans l'exercice de la déclamation, qui entraîne l'élève à plaider le pour et le contre d'un cas donné, il permet de réfléchir aux arguments de l'une et l'autre partie en les juxtaposant. On le trouve à différents endroits du discours, où il sert tantôt à informer, tantôt à argumenter, tantôt encore à susciter l'indignation ou la pitié. Parfois, il se déploie ensemble contre ensemble pour imposer un point de vue, parfois il se présente comme un procédé stylistique qui souligne une opposition de façon plus ramassée, à la fois sonore et visuelle. Les critères auxquels il fait appel pour révéler le pire ou le meilleur varient également, qu'il s'agisse de valeurs, comme l'honnêteté, la dignité, la justice, le sacré, ou de notions plus concrètes, comme l'utilité, la nécessité ou la temporalité. 


\section{NOTES}

1. Voir récemment F. Berardi, La retorica degli esercizi preparatori. Glossario ragionato dei progymnásmata, Zürich-New York, Olms, 2017 ; P. Chiron, Manuel de rhétorique ou Comment faire de l'élève un citoyen, Paris, Belles Lettres, 2018 ; P. Chiron, B. Sans éd., Les progymnasmata en pratique de l'Antiquité à nos jours, Paris, Rue d'Ulm, 2020.

2. D. van Mal-Maeder, «Des progymnasmata à la déclamation : entre hier et aujourd'hui », dans P. Chiron, B. Sans, op. cit., p. 118-131.

3. Cf. déjà Isocrate, Panathénaïque, 39-41; Aristote, Rhétorique, 1368a ; Pline, Panégyrique de Trajan, 53,1: «nihil non parum grate sine comparatione laudatur » (« il n'y a pas d'éloge convenable sans comparaison ») ; voir L. Pernot, La rhétorique de l'éloge dans le monde gréco-romain, Paris, Institut d'Études Augustiniennes, 1993, p. 690-698.

4. M. Patillon (collab. G. Bolognesi), Aelius Théon. Progymnasmata, Paris, Belles Lettres, $2002^{2}$ (1997 $)$, p. LXXXI ; M. Patillon, Corpus rhetoricum. Anonyme, Préambule à la rhétorique; Aphthonios, Progymnasmata ; Pseudo-Hermogène, Progymnasmata, Paris, Belles Lettres, 2008, p. 86-88.

5. F. Focke, "Synkrisis ", Hermes, 58, 1923, p. 327-368; voir aussi O. Hense, Die Synkrisis in der Antiken Literatur, Freiburg, Prorectoratsrede, 1893; J. Bompaire, Lucien écrivain. Imitation et création, Paris, De Boccard, 1958, p. 271-274 ; 289-291 ; L. Pernot, op. cit.

6. Quintilien, Institution oratoire, $\mathrm{X}, 1$; Tacite, Dialogue des orateurs, 5-13 ; Aulu-Gelle, Nuits attiques, IX, 9.

7. Sénèque le Père, Controverses, I, praef. 1, qui emploie le verbe iudicare, « juger ».

8. Sénèque le Père, Controverses, IV, praef. 4-6. Cette anecdote, construite sur un parallèle, n'a rien d'une digression: voir D. van Mal-Maeder, «Controversial games: didactical voices and the construction of discourse in Seneca's Controversiae and Suasoriae " dans M. Dinter, Ch. Guérin, M. Martinho éd., Reading Roman Declamation. Seneca the Elder, Oxford, OUP, 2020, p. 318-331.

9. Quintilien, Institution oratoire, II, 4, 21, éd. et trad. J. Cousin, Paris, Belles Lettres, 1976.

10. Cicéron, De l'invention, I, 17, éd. et trad. G. Achard, Paris, Belles Lettres, 2002 ; voir H. Lausberg, Handbook of Literary Rhetoric. A Foundation for Literary Study, Leyde-Boston-Cologne, Brill, 1998 (trad. anglaise de Handbuch der literarischen Rhetorik. Eine Grundlegung der Literaturwissenschaft, $1973^{2}$ ), p. 76-77, § 181-182.

11. Quintilien, Institution oratoire, III, 10, 3, éd. et trad. J. Cousin, Paris, Belles Lettres, 1976.

12. M. Winterbottom, The Minor Declamations ascribed to Quintilian, Berlin-New York, De Gruyter, 1984 ; C. Oppliger, «Quelques réflexions sur la méthode (ou les méthodes ?) du Maître des Petites déclamations ", dans A. Casamento, D. van Mal-Maeder, L. Pasetti éd., Le Declamazioni minori dello Pseudo-Quintiliano. Discorsi immaginari tra letteratura e diritto, Berlin-Boston, De Gruyter, 2016, p. 103-116 ; M. Winterbottom, « The Words of the Master », Maia, 70, 2018, p. 73-83 ; L. Pasetti et al., Le Declamazioni minori attribuite a Quintiliano I (244-292). Testo, traduzione e commento, Bologne, Pàtron, 2019, en part. p. XI-XXXV, n'exclut pas que Quintilien soit l'auteur de ce manuel.

13. Ps.-Quintilien, Déclamations mineures, 268, éd. M. Winterbottom, op. cit. (pour ces textes, la traduction est mienne); voir les commentaires de M. Winterbottom, op.cit., p. 358-359 et L. Pasetti et al., op.cit., p. 351-352 ad loc. On trouve un sujet quasi identique chez Quintilien, Institution oratoire, VII, 1, 38.

14. E. Berti, « Una declamazione per burla. Il Iudicium coci et pistoris di Vespa (Anth. Lat. 199 R. = 190 Shackleton Bailey)», Maia, 70, 2018, p. 163-182.

15. L'utilité de l'art oratoire est affirmée par Aristote, Rhétorique, 1355a, 20-21; Quintilien, Institution oratoire, II, 16 ; Tacite, Dialogue des orateurs, 5.

16. Quintilien, Institution oratoire, VII, 2, 25, éd. et trad. J. Cousin, Paris, Belles Lettres, 1977. 
17. Ps.-Quintilien, Déclamations mineures, 288, 1 : « Tyrrannicida optet quod uolet » (« Un tyrannicide pourra demander ce qu'il voudra »); 282 : «Tyrannicidae praemium» («La récompense due au tyrannicide »), voir L. Pasetti et al., op. cit., p. 430-431; T. Wycisk, Quidquid in foro fieri potest. Studien zum römischen Recht bei Quintilian, Berlin, Duncker \& Humblot, 2008, p. 200-208. Sur la figure du tyran et la législation qui l'entoure, voir encore L. Pasetti et al., op. cit., p. 262 et p. 345 ; R. Tabacco, Il tiranno nelle declamazioni di scuola in lingua latina, Turin, Academia delle scienze, 1985.

18. Quintilien, Institution oratoire, VII, 4, 21 ; cette question se pose aussi, de façon quelque peu paradoxale, à propos de délateurs : cf. Quintilien, Institution oratoire, III, 10, $3:$ « inter delatores, uter praemium meruerit » (« entre deux délateurs, lequel mérite la récompense »).

19. Pour les personnages $d u$ riche et du pauvre, $c f$. B. Santorelli, [Quintiliano]. Il ricco accusato di tradimento (Declamazioni maggiori, 11), Gli amici garanti (Declamazioni maggiori, 16), Cassino, Edizioni Università di Cassino, 2014.

20. Ps.-Quintilien, Déclamations mineures, 345, 9.

21. Ps.-Quintilien, Déclamations mineures, 258 et 371 : «Vir fortis optet quod uolet » («Un héros de guerre pourra réclamer ce qu'il voudra »); Sénèque le Père, Controverses, X, 2: «Vir fortis quod uolet praemium optet » («Un héros de guerre pourra réclamer la récompense de son choix »); Ps.Quintilien, Déclamations mineures, 293 et 304: «Viro forti praemium» («La récompense due au héros de guerre »); $c f$. Quintilien, Institution oratoire, VII, 1, 24 ; VII, 5, 4; VII, 7, 4, etc. ; voir T. Wycisk, op. cit., p. 215-221. Sur la figure du uir fortis, cf. M. Lentano, L'eroe va a scuola, La figura del uir fortis nella declamazione latina, Naples, Loffredo, 1998.

22. Ps.-Quintilien, Déclamations mineures, 258, 5 ; voir L. Pasetti et al., op. cit, p. 292 ad loc. Pour un cas similaire, $c f$. Sénèque le Père, Controverses, VIII, 5 , où on trouve ce parallèle $(\S 2)$ : «Ille annos suos exercuit, ego uici meos; tu fregisti bellum, ego sustuli» («Lui a exercé son âge, moi je l'ai vaincu ; toi, tu as pulvérisé cette guerre, moi je l'ai soufferte »); $c f$. aussi ibidem, X, 2, où la loi exige de trancher entre deux requérants non pas par les armes, mais par un procès : «Vir fortis quod uolet praemium optet; si plures erunt, iudicio contendant » (« Un héros de guerre pourra demander ce qu'il voudra; si deux hommes ou plus ont combattu héroïquement, ils se disputeront la récompense en justice »). Cette variante amène une comparaison en faveur du fils : cf. X, 2, 11: "Miraris si patri hac lege subducitur qui ei et comparatur et praefertur? " ("Tu t'étonnes si cette loi soustrait à l'autorité de son père un fils qu'elle met en parallèle avec lui et place au-dessus de lui ?»). Voir A. Casamento, «Come un figlio: variazioni tematiche e modalità narrative. A propositio di Sen. contr. 10, 2 e decl. min. 258 ", dans A. Casamento, D. van Mal-Maeder, L. Pasetti, op. cit., p. 191-212; L. Landolfi, "Sulle tracce di Ovidio epico? Conteste tra padre e figli in Ps. Quint. 258 », Maia, 70, 2018, p. 98-117, compare la Petite déclamation 258 avec le jugement des armes chez Ovide, Métamorphoses, XIII, 1-383.

23. Ps.-Quintilien, Déclamations mineures, $278,8$.

24. Voir L. Pasetti et al., op. cit. p. 410 ad loc.; F. Citti, L. Pasetti, « Declamazione e stilistica » dans M. Lentano éd., La declamazione latina. Prospettive a confronto sulla retorica di scuola a Roma antica, Naples, Liguori Editore, 2015, p. 115-148.

25. Quintilien, Institution oratoire, VII, 7, 3. La même problématique vaut pour la loi sur les femmes violées, qui autorise la victime d'un viol de choisir entre faire condamner à mort son agresseur ou l'obliger à l'épouser sans dot («Rapta raptoris aut mortem aut indotatas nuptias optet »); dans le cas où un homme viole deux femmes la même nuit et qu'elles optent l'une pour le mariage, l'autre pour la mort (c'est le cas étudié par Sénèque le Père, Controverses, I, 5), une comparaison s'impose.

26. Cicéron, De l'invention, II, 144-145, éd. et trad. G. Achard, Paris, Belles Lettres, 2002. Il existe différentes versions de la mort de ce tyran, e.g. Xénophon, Helléniques, VI, 4, 35-37; Cicéron, Des devoirs, II, 7, 25 ; Plutarque, Pélopidas, 35 ; Valère Maxime, Faits et dires mémorables, IX, 13, ext. 3. Sur les lois contradictoires, $c f$. encore Rhétorique à Herennius, I, 20 ; II, 15 ; Quintilien, Institution 
oratoire, VII, 7 ; L. Calboli Montefusco, La dottrina delle status nella retorica greca e romana, Bologne, Olms-Weidmann, 1986, p. 166-178.

27. G. Dimatteo, Audiatur et altera pars. I discorsi doppi nelle Declamationes minores in Calpurnio Flacco, Bologne, Pàtron Editore, 2019, p. 29-41 ; L. Pasetti et al., op. cit., p. 384-385.

28. Ps.-Quintilien, Déclamations mineures, $274,1$.

29. Ibidem, 274, 3-4.

30. Ibidem, 274, 11.

31. Ibidem, 324, 1.

32. Voir B. Santorelli, A. Stramaglia, [Quintiliano]. Il muro con le impronte di una mano (Declamazioni maggiori, 1), Cassino, Edizioni Università di Cassino, 2017 ; J. Pingoud, A. Rolle, Déclamations et intertextualité. Discours d'école en dialogue, Berne, Peter Lang, 2020, p. 208-238, d'où est tirée la traduction des passages que je cite.

33. Quintilien, Institution oratoire, VII, 2, 9-10; III, 10, 4; voir G. Krapinger, A. Stramaglia, [Quintilian]. Der Blinde auf der Türschwelle (Grössere Deklamationen, 2), Cassino, Edizioni Università di Cassino, 2015, p. 48-52 à propos de Ps.-Quintilien, Déclamations majeures, II, qui présente un sujet très similaire et contient aussi une antikategoria.

34. Ps.-Quintilien, Déclamations majeures, I, 13,2: "Transeo illum uulgarem et omnibus notum de conparatione personarum locum» ("Je laisse de côté le lieu commun de la confrontation des caractères : il est bien connu de tous »): voir B. Santorelli, A. Stramaglia, op.cit., p. 141-142, n. 223.

35. Ce découpage est annoncé en I, 5, 4: «Causam igitur miserrimi adulescentis sic apud uos agere proposui, ut primum ipsum defendam, quasi reus tantum sit; deinde, cum esse securus de huius innocentia coepero, tunc ingrediar nouercae accusationem » («J'ai donc décidé de plaider devant vous la cause de ce malheureux garçon de la manière suivante : en premier lieu, je le défendrai comme s'il était un simple accusé ; puis, quand je me sentirai un peu rassuré d'avoir prouvé son innocence, alors j'entreprendrai d'accuser la belle-mère ») ; cf. Ps.-Quintilien, Déclamations majeures, II, 16, 1-3 : «Tractemus nunc, iudices, ipsius sceleris comparationem. Caecus ignorat ubi iaceat senex, an iam quiescat... Tu sentis quando senem uicerit lassitudo curarum» («Passons maintenant, Juges, à la comparaison des circonstances du crime. L'aveugle ignore où le vieil homme est couché, s'il dort déjà... Toi tu sens quand le poids des soucis a eu raison de lui ») : voir G. Krapinger, A. Stramaglia, op. cit., p. 198-199 n. 323 et p. 201-202, n. 333-334.

36. Ps.-Quintilien, Déclamations majeures, I, 16, 6-1, 17, 2.

37. Pour cette déclamation, je me permets de renvoyer à D.van Mal-Maeder, [Quintilien]. Le malade racheté (Grandes déclamations, 5), Cassino, Edizioni Università di Cassino, 2018, dont je reprends ici la traduction.

38. Selon les recommandations de Quintilien, Institution oratoire, IV, 2,52 : «Credibilis autem erit narratio... si personas conuenientes iis, quae facta credi uolemus, constituerimus » (« La narration sera particulièrement plausible... si nous campons des caractères en harmonie avec les faits que nous voulons faire admettre »), éd. et trad. J. Cousin, Paris, Belles Lettres, 2003.

39. Ps.-Quintilien, Déclamations majeures, $\mathrm{V}, 3,1$.

40. D. van Mal-Maeder, op. cit. (2018), p. 121-122, n. 43.

41. D. van Mal-Maeder, op. cit. (2018), p. 186-187, n. 288 et p. 188-189, n. 291.

42. Ps.-Quintilien, Déclamations majeures, V, 16, 5-17, 1 .

43. Dans un passage traitant de la question de savoir si la comparatio est une figure de mots ou de pensée, Quintilien cite un passage du discours Pour Muréna de Cicéron où l'on observe le même type d'antithèse (Institution oratoire, IX, 2, 100-101): "Vigilas tu de nocte ut tuis consultoribus respondeas, ille ut eo quo contendit mature cum exercitu perueniat; te gallorum, illum bucinarum cantus exsuscitat » («La nuit, vous veillez, toi, pour répondre à ceux qui te consultent, lui pour arriver à temps avec son armée au point fixé ; toi, ce sont les accents du coq qui te réveillent, lui, ceux des buccins »); sur ce passage controversé, voir A.Cavarzere, L.Cristante, M. Fabi Quintiliani, 
Institutionis oratoriae liber IX. Introduzione, testo, traduzione e commento, I, Hildesheim, Weidmann, 2019, p. 469-471.

44. Ps.-Quintilien, Déclamations majeures, V, 1,5: «Sola, quid facerem, necessitas, sola iuuenum meorum aduersa suaserunt » ("Seule la nécessité, seule la détresse de mes garçons m’ont persuadé de ce que je devais faire»), avec D. van Mal-Maeder, op.cit., p. 113-114, n. 26 ad loc.; V, 6, 1 ; $\mathrm{V}, 11,4 ; \mathrm{V}, 19,1:$ : Ponere uos, iudices, uelut in illa necessitatis meae praesentia uolo " (« Messieurs les Juges, je veux vous placer, pour ainsi dire, devant la nécessité à laquelle je faisais face »).

45. Ps.-Quintilien, Déclamations majeures, V, 5, 3: «Explicuit iustitiam comparationis, qui decessit etiam redemptus, et in perituro filio nihil aliud electum est » (« En rendant l'âme alors même qu'il avait été racheté, il a démontré que ma décision était juste $») ; \mathrm{V}, 13,1$ : Quantum intellego, iudices, filius, cui profuturum non erat ut eligerem, hoc solum ferre non potest, quod redemptus est frater ( D'après ce que je comprends, Messieurs les Juges, mon fils, auquel il ne devait pas être utile que je le choisisse, ne peut tout simplement pas supporter que son frère ait été racheté »); $f$. Cicéron, De l'invention, I, 15 : «Conparatio est cum aliud aliquid factum rectum aut utile contenditur, quod ut fieret, illud quod arguitur dicitur esse commissum» («On emploie l'alternative quand on plaide que l'on a réalisé quelque autre action juste et avantageuse et que c'est pour la réaliser que l'on a commis l'action qui est reprochée »), éd. et trad. G. Achard, Paris, Belles Lettres, 2002.

46. Th. Zinsmaier, [Quintilian]. Die Hände der blinden Mutter (Grössere Deklamationen, 6), Cassino, Edizioni Università di Cassino, 2009, p. 62 et p. 228-229, n. 279. On trouve un sujet très proche chez Sénèque le Père, pour lequel Latron développa une argumentation essentiellement fondée sur la comparaison (cf. Controverses, VII, 4, 3).

47. Ps.-Quintilien, Déclamations majeures, VI, 14, 6 (ma traduction).

48. Ibidem, VI, 15, 1 : «Non conparabo personas, quamuis apud omnes gentes plus iuris habeat pater » ( Je ne vais pas comparer nos rôles, même si, chez tous les peuples du monde, un père a plus de droits ») : voir Th. Zismaier, op. cit., p. 226-227, n. 274 ad loc.

49. Ps.-Quintilien, Déclamations majeures, VI, 15, 3-4.

50. Ps.-Quintilien, Déclamations majeures, VI, 17, 2 ; voir Th. Zinsmaier, op. cit., p. 232, n. 290.

51. Ps.-Quintilien, Déclamations majeures, VI, 17, 4 - VI, 18, 6 ; voir Th. Zinsmaier, op. cit., p. 234, n. 295 et 300 ; comparer Sénèque le Père, Controverses, VII, 4, 5 (Varius Geminus) : " At tu caeca es : ille (sc. pater) hoc infelicior, quod uidet. Quid enim? Videt catenas suas et caedes et uolnera et cruces eorum, qui non redimuntur » (« Toi, tu es aveugle : lui [le père] est d'autant plus malheureux qu'il voit. Tu sais pourquoi ? Il voit ses chaînes, les meurtres, les blessures et les croix de ceux qu'on ne rachète pas »).

52. Ps.-Quintilien, Déclamations majeures, VI, 18, 6.

53. Ps.-Quintilien, Déclamations majeures, VI, 19, 2 ; sur cette figure de style, fréquente dans les déclamations, voir F. Citti, L. Pasetti, op. cit., p. 122.

54. E.g. Ps.-Quintilien, Déclamations majeures, IX, 22, 2-3, avec G. Krapinger, [Quintilian]. Der Gladiator (Grössere Deklamationen, 9), Cassino, Edizioni Università di Cassino, 2007, p. 167-169, n. 413-419; cf. Quintilien, Institution oratoire, V, 10, 87 : «Adposita uel comparatiua dicuntur, quae minora ex maioribus, maiora ex minoribus, paria ex paribus probant» («On appelle arguments de voisinage ou de comparaison ceux qui vont du plus grand au plus petit, du plus petit au plus grand, du même au même »); VIII, 4,9-12; H. Lausberg, op. cit. p.191, §404. Ce procédé est fréquent en poésie; dans ses lettres d'exil, par exemple, Ovide ne cesse de se comparer à Ulysse, en se lamentant de ce que ses propres souffrances surpassent celles du héros de l'Odyssée, e.g. Ovide, Tristes, I, 5, 59-62. Voir, dans l'Atelier de cette présente livraison, le chapitre de Gérard Pelletier portant sur le « Locus a comparatione maiorum, minorum, parium ».

55. Ps.-Quintilien, Déclamations majeures, VI, 21, 7.

56. Voir Th. Zinsmaier, op. cit., p. 244-245, n. 347 et 348 ; l'exemple de Cimon comme modèle de piété filiale se retrouve ailleurs dans le corpus déclamatoire et forme même le sujet d'une controverse : $c f$. Sénèque le Père, Controverses, IX, 1 ; Quintilien, Déclamations mineures, 302, 5. 


\section{AUTEUR}

\section{DANIELLE VAN MAL-MAEDER}

Université de Lausanne 